NOTE TECHNIQUE

\title{
MARQUAGE D'OMBLES CHEVALIER (SALVELINUS ALPINUS) DE PETITE TAILLE PAR INJECTION DE MICROMARQUES MAGNÉTISÉES
}

\author{
A. CHAMPIgNEULLE \\ avec la collaboration technique de J. ESCOMEL et Ph. LAURENT \\ Station d'Hydrobiologie Lacustre, INRA - B.P. $11 \mathrm{~F}$ - \\ 75, avenue de Corzent - 74203 THONON LES BAINS Cedex, France
}

Recu le 17 Octobre 1986

Received 17 October, 1986

Accepté le 26 Janvier 1987

Accepted 26 January, 1987

\begin{abstract}
RESUMÉ
La technique de marquage de poissons par injection, dans le cartilage nasal, de micromarques magnétisées codées est présentée. Ce type de marquage a été testé avec succès sur des ombles chevalier (Sa/velinus alpinus) de très petite taille $(22-55 \mathrm{~mm})$. Quand la technique est bien maîtrisée, le taux de rétention des marques est élevé $(\geq 98 \%)$. La vitesse de marquage a été de 250300 /personne/heure pour des ombles de 2-3 cm et de 400-450/personne et par heure pour ceux de 4-5 cm. La mortalité au cours du marquage est très faible. La survie et la croissance, évaluées après un mois, en conditions d'élevage, n'ont pas été significativement affectées par le marquage magnétique. Des recommandations sont faites.
\end{abstract}

\section{MARKING SMALL ARCTIC CHARS(SALVELINUS ALPINUS) BY INJECTION OF MAGNETIZED MICROTAGS}

\section{SUMMARY}

The paper presents the technique of marking fish by injection of magnetized coded microtags in the nose. The technique has been successfully used on small (22-55 $\mathrm{mm}$ total length) arctic chars (Salvelinus alpinus $L$.). When the technique is well controlled, tag retention is high ( $\geq 98 \%$ ). Observed tagging rate was 250-300/person/hour for char with a size of 2-3 cm and 400/500/person/hour at a size of $4-5 \mathrm{~cm}$. Mortality during tagging was very low. Survival and growth, measured after one month of rearing, were not significantly affected by microtagging. Some recommendations are given.

\section{INTRODUCTION}

Un article récent (CHAMPIGNEULLE et ESCOMEL, 1984) a mis en évidence la possibilité de marquer définitivement des salmonidés de petite taille $(3-5 \mathrm{~cm})$ par cautérisation de la nageoire adipeuse ou ablation des deux nageoires pelviennes. Néanmoins la technique d'ablation de nageoires ne permet pas de différencier beaucoup de lots sans introduire de biais liés à la nature de la (ou des) nageoires sectionnées.

L'utilisation de la technique de marquage à l'aide de micromarques codées, injectées dans le cartilage nasal puis magnétisées, se développe à l'étranger (LEARY et MURPHY, 1975; HARACHE et al., 1978; OPDYKE et ZAJAC, 1981 ; THROWER et SMOKER, 1984 ; HARGREAVES et LEBRASSEUR, 1986 ; MENG et al., 1986 ; SALOJARVI, 1986). La technique commence à être utilisée en France par le C.S.P. (Conseil Supérieur de la Pêche) en Bretagne sur des smolts de saumon atlantique (Salmo salar L. ) et par l'INRA sur des alevins de salmonidés: truite (Salmo trutta L.), omble chevalier (Sa/velinus alpinus L.) et corégone (Coregonus sp.). Le but de la présente note est de présenter les premiers résultats expérimentaux sur l'utilisation du marquage magnétique dans le cas d'ombles de très petite taille $(2,2-5,5 \mathrm{~cm})$ : mise en œuvre, influence sur la survie et la croissance, contrôle des pertes de marques.

\section{I - MATÉRIEL ET MÉTHODES}

\section{Présentation générale de la technique de marquage magnétique}

Le marquage magnétique a été testé sur des alevins d'omble chevalier élevés sur granulés à truite à la pisciculture expérimentale de la Station d'Hydrobiologie Lacustre, INRA, à Thonon les Bains. Au moment des essais, la température de l'eau a varié entre 11 et $13^{\circ} \mathrm{C}$. 
Le marquage a été pratiqué à l'aide du système Northwest Marine Technology (N.M.T., fig 1a) avec la version MK II permettant l'injection de demi-marques codées (longueur: 0,5 mm: largeur : $0,25 \mathrm{~mm}$ ) dans le cartilage nasal (fig. $1 \mathrm{~b}$ ). Les essais $n$ 'ont pas porté sur la récupération des marques. Notons cependant que les poissons marqués sont repérés en les passant devant un détecteur de terrain (système NMT, fig. 1c) qui émet un signal sonore en présence de la marque. Un système de codage par encoches permet d'identifier $\mathbf{4 0 9 6}$ lots différents. La lecture du code, réalisée sous binoculaire, n'est possible qu'après extraction de la marque, ce qui implique l'abattage du poisson. Il existe cependant un modèle de marque, lisible aux rayons $X$, permettant le décodage sur des poissons vivants.

a) $\rightarrow$

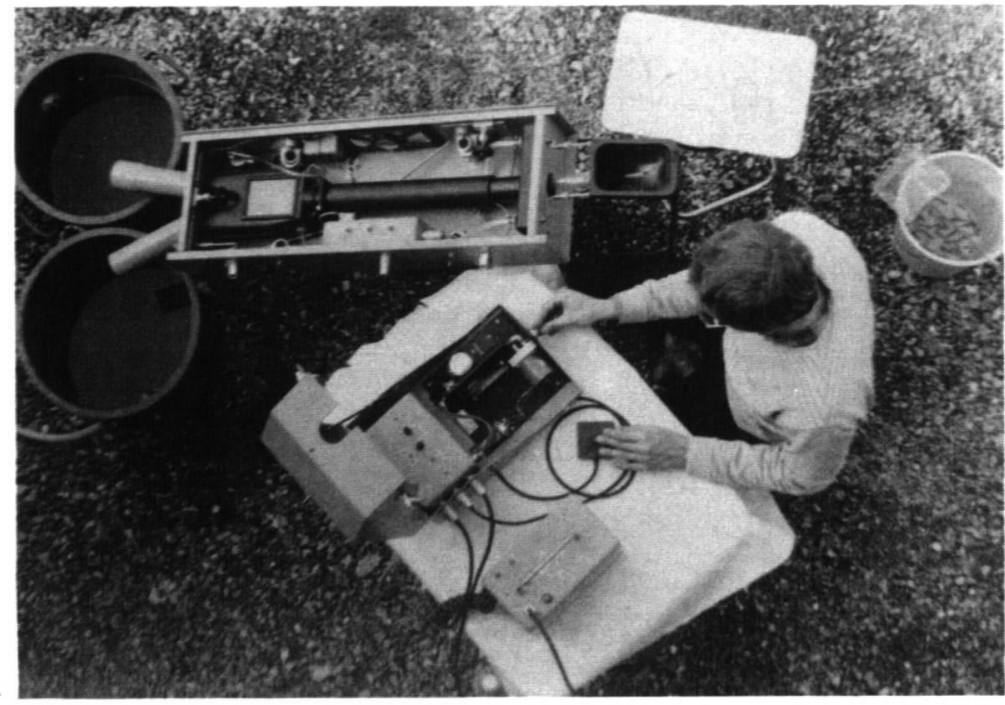

- b)
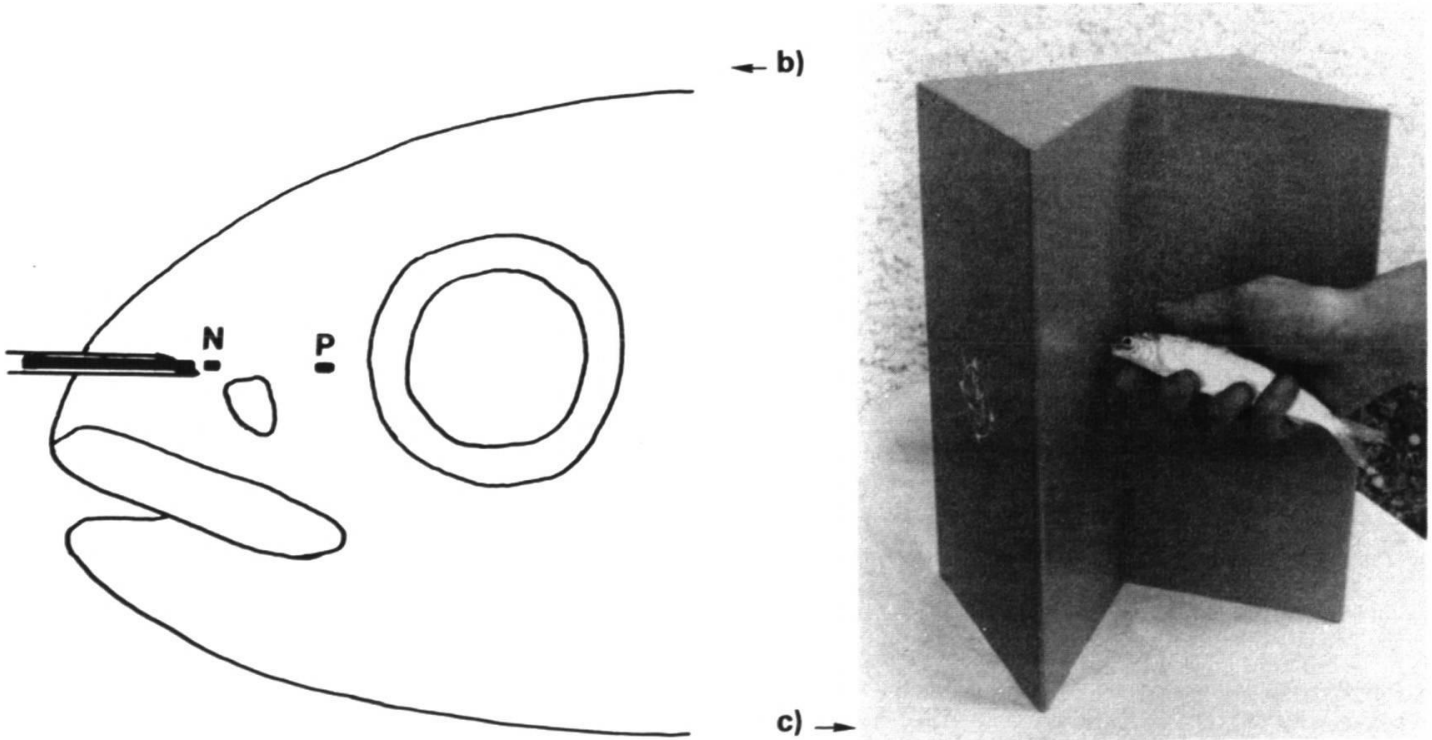

Figure 1 : Equipement de marquage magnétique (Northwest Marine Technology): a) injection de marque et systême assurant la magnétisation. le contrôle du marquage et le comptage.

b) implantation des marques ( $N$ : position normale : $\mathbf{P}$ : position profonde).

c) détecteur de terrain pour les marques magnétisées.

Figure 1 : Magnetic tagging equipment (Northwest Marine Technology) :

a) tag injector and quality control device

b) tag implantation ( $N$ : usual implantation depth ; $P$ : deep implantation depth).

c) sampling field detector. 


\section{a. OPERATIONS PRÉLIMINAIRES}

- Isolement d'un lot homogène en taille.

- Choix d'un embout préfabriqué adapté à la taille et à la forme de la tête d'un individu moyen. Au besoin fabrication d'un embout en résine par moulage sur un poisson représentatif du lot.

- Aiguisage de la pointe de l'aiguille.

- Réglage en position terminale de la marque dans l'aiguille en bout de course.

- Essais de marquage sur quelques poissons et contrôle de la profondeur d'implantation par dissection

- Choix de la profondeur d'implantation en fonction de la taille du poisson et de l'obtention d'un très bon taux de rétention initiale détecté grâce aú contrôleur de qualité.

\section{a. PRELIMINARY OPERATIONS}

- sorting to homogenize the size of fish.

- choice of a standard head mould adapted to the size and head shape of a representative specimen. If necessary a special head mould can be prepared by a resin casting.

- sharpening the needle.

- positioning the tag just behind the tip of the needle when the needle is at the outermost end of its travel.

- verifying the position ot the tag by dissecting the skull and looking for the tag.

- choice of an implantation depth adapted to the size and head shape of fish and giving a high retention rate controlled with the quality control device.

\section{b. MARQUAGE DU LOT}

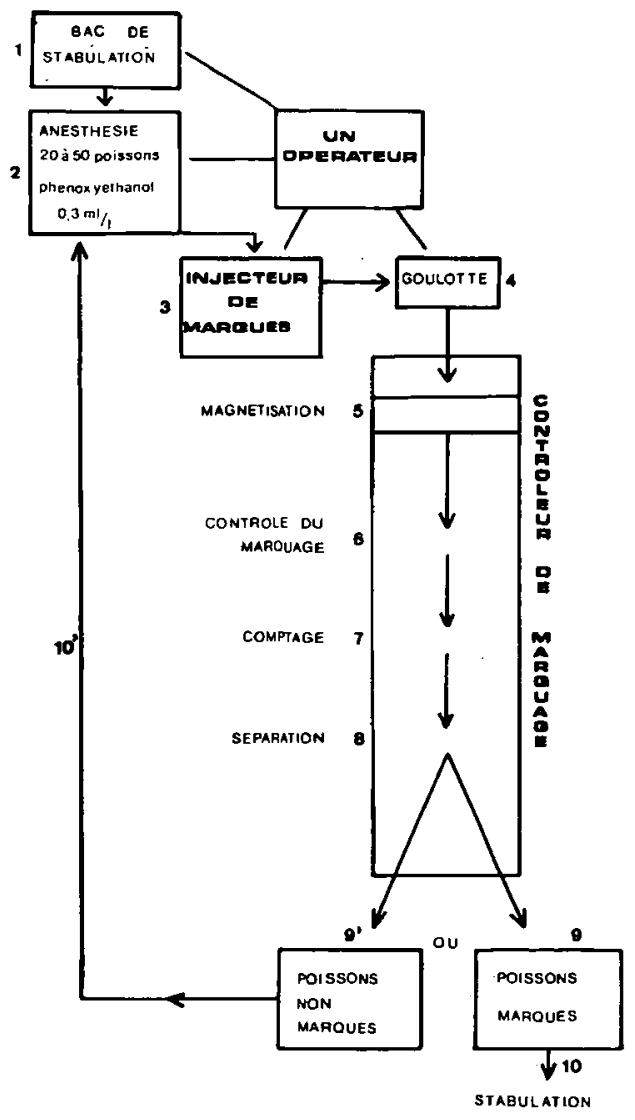

Figure 2 : Principales étapes du marquage magnétique d'un lot de poissons.

a) opérations préliminaires

b) marquage du lot.

Figure 2 : Main steps of the marking of a batch of fish with magnetized microtags.

a) preliminary operations

b) tagging operations. 
Les différentes étapes du marquage d'un lot de poissons sont décrites dans la figure 2. L'ensemble de ces opérations est réalisable par une seule personne. Les plus petits individus ( $L \leq 35 \mathrm{~mm}$ ) sont prélevés dans le bac d'anesthésie à l'aide d'une palette puis repris à la main à partir de la palette. Les plus gros $(\mathrm{L} \geq 35 \mathrm{~mm})$ sont directement prélevés à la main à partir du bac d'anesthésiant. Les poissons sont ensuite portés, un à un, dans l'embout qui permet le positionnement de leur tête au moment de l'injection de la marque. L'injection est réalisée automatiquement grâce à une aiguille creuse traversant l'embout. Après pénétration de l'aiguille, une marque est poussée à l'intérieur de celle-ci (fig. 1 b) et l'aiguille se rétracte. Habituellement les marques sont implantées dans le cartilage nasal, juste avant des cavités olfactives (position $\mathrm{N}$, fig $1 \mathrm{~b}$ ).

\section{Essais de marquage et suivi en pisciculture}

\subsection{Essais de 1985}

Deux groupes d'ombles ( $\mathrm{A}$ et $\mathrm{B}$ ) différents par la taille des poissons ont été marqués. Pour chacun des deux groupes, un échantillon de 100 individus a été mesuré, puis, quatre lots de 300 poissons ont été constitués (tab 1):

1. adipeuse cautérisée $(\operatorname{ad} \emptyset)$

2. marque magnétique (1/2 M)

3. témoin non marqué (non ${ }^{*}$ )

4. marquage magnétique et cautérisation de l'adipeuse (ad ø et $1 / 2 \mathrm{M}$ )

\begin{tabular}{|c|c|c|c|c|c|c|c|c|}
\hline $\begin{array}{l}\text { Groupe } \\
\text { (année) }\end{array}$ & Période & $\begin{array}{c}\text { Bassin } \\
n^{\circ}\end{array}$ & Lot & Marquage & $\begin{array}{l}\overline{\mathrm{L}}+\mathrm{S} . \mathrm{D} . \\
\text { à } 95 \%\end{array}$ & $\begin{array}{l}P \\
\text { (fog) }\end{array}$ & $\begin{array}{l}5 \\
(x)\end{array}$ & $\begin{array}{l}R \\
(\%)\end{array}$ \\
\hline \multirow{3}{*}{$(1985)$} & Début & - & $=$ & 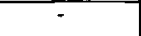 & $25,5 \div 0,5$ & 177 & - & - \\
\hline & \multirow{2}{*}{$\begin{array}{l}\text { après } \\
1 \\
\text { mois }\end{array}$} & 1 & $\begin{array}{l}\text { A1 } \\
\text { A2 }\end{array}$ & $\begin{array}{l}\text { ad } \\
1 / 2\end{array}$ & $\begin{array}{r}49,4 \pm 0,7 \\
49,6 \pm 0,6 \\
(50,5 \pm 1,5)\end{array}$ & $\begin{array}{l}300 \\
360\end{array}$ & $\begin{array}{l}96,3 \\
96,0\end{array}$ & $\overline{18,1}$ \\
\hline & & 2 & $\begin{array}{l}A 3 \\
A 4\end{array}$ & $\begin{array}{l}\text { non * } \\
1 / 2 M+\text { adpo }\end{array}$ & $\begin{array}{c}46,2 \geq 0,6 \\
48,4 \pm 0,6 \\
(47,2 \geq 1,4)\end{array}$ & $\begin{array}{r}820 \\
1100\end{array}$ & $\begin{array}{l}88,3 \\
82,0\end{array}$ & $\overline{17,9}$ \\
\hline \multirow{3}{*}{$\begin{array}{c}\text { B } \\
(1985)\end{array}$} & Début & - & - & - & $46.6 \pm 1.6$ & 900 & - & - \\
\hline & \multirow{2}{*}{$\begin{array}{c}\text { après } \\
\text { mois }\end{array}$} & 3 & $\begin{array}{l}\text { B1 } \\
\text { B2 }\end{array}$ & $\begin{array}{l}\text { ad } \\
1 / 2 \mathrm{H}\end{array}$ & $\begin{array}{l}66,3 \pm 0,7 \\
67,3 \pm 0,7\end{array}$ & $\begin{array}{l}2600 \\
2900\end{array}$ & $\begin{array}{l}93,7 \\
95,0\end{array}$ & 69.5 \\
\hline & & 4 & $\begin{array}{l}83 \\
84\end{array}$ & $\frac{\text { non }}{1 / 2 M+\text { ad } \sigma}$ & $\begin{array}{l}69,4 \pm 0.6 \\
69,1 \pm 0.6 \\
(69,4 \pm 0.6)\end{array}$ & 3100 & $\begin{array}{l}93,3 \\
92,0\end{array}$ & 79.7 \\
\hline \multirow{6}{*}{$A^{\prime}$} & Début & - & - & - & $28,2 \pm 0,4$ & 205 & 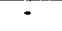 & - \\
\hline & \multirow{5}{*}{$\begin{array}{c}\text { après } \\
1 \\
\text { mois }\end{array}$} & 1 & $\begin{array}{l}\text { A'1 } \\
\text { A'2 }\end{array}$ & non - & $\begin{array}{l}39,2 \pm 0,4 \\
39,2 \pm 0,4\end{array}$ & $\begin{array}{l}520 \\
537\end{array}$ & $\begin{array}{l}98,0 \\
94.7\end{array}$ & - \\
\hline & & 3 & $A^{\prime} 3$ & non * & $40,6 \pm 0.4$ & 603 & 96,3 & - \\
\hline & & 4 & $A^{\prime} 4$ & $1 / 2 \mathrm{M}$ & $\begin{aligned} 40,3 & =0,4 \\
(40,2 & \pm 0,3)\end{aligned}$ & 561 & 98.3 & 98,0 \\
\hline & & 5 & $A^{\prime} 5$ & $1 / 2 \mathrm{M}$ & $\begin{array}{l}40,0 \pm 0,4 \\
(40.0 \pm 0.4)\end{array}$ & 549 & 96.7 & 98,3 \\
\hline & & 6 & A' 6 & $1 / 2 \mathrm{M}$ & $\begin{aligned} 39,5 & \pm 0,4 \\
(39,5 & \pm 0,4)\end{aligned}$ & 518 & 97,0 & 98,3 \\
\hline
\end{tabular}

Tableau 1 : Influence du marquage sur la croissance et la survie après 1 mois d'élevage. (ad $\phi)$ : adipeuse cautérisée: $(1 / 2 \mathrm{M}): 1 / 2$ marque magnétique: (non ${ }^{*}$ : non marqué ; (ad + $1 / 2 \mathrm{M})$ : adipeuse cautérisée et demi-marque magnétique. ( $L \pm$ SD): longueur moyenne des survivants \pm écart standard.

Pour les lots marqués magnétiquement: L est aussi calculée sur les survivants ayant retenu la marque (valeur entre parenthèses)

(P): poids moyen en $\mathrm{mg}$; (S \%) : taux de survie

( $R \%$ ) : pourcentage de survivants ayant retenu la marque.

Table 1 : Effect of tagging on growth and survival after one month of rearing.

(ad $\phi)$ : burned adipose fin; $(1 / 2 \mathrm{M})$ : half-length tag injected; (non*): no tagging: (ad $\phi+1 / 2 M)$ : burned adipose fin and half-length tag injected.

( $L \pm$ S.D.): average length of survivors \pm standard deviation.

For batches identified by microtags: $L$ was also calculated for survivors which have not lost their tags (figure in brackets).

(P): mean weigth, in $\mathrm{mg}$; (S\%) : survival rate

(R\%) : percentage of survivors which retained the microtag. 
Le marquage magnétique a été pratiqué comme indiqué ci-dessus. L'ablation de l'adipeuse a été réalisée par cautérisation selon la technique décrite par CHAMPIGNEULLE et ESCOMEL (1984). Après marquage, les lots expérimentaux ont été élevés en auges de pisciculture pendant 1 mois. Pour chacun des groupes, les lots 1 et 2 ont été placés dans le même bac et les lots 3 et 4 ensemble dans un autre bac. L'objectif était en effet de comparer d'une part marquage magnétique et cautérisation de l'adipeuse et, d'autre part, de comparer les résultats entre les témoins non marqués et les individus à la fois marqués magnétiquement et par cautérisation de l'adipeuse. Après 1 mois les survivants de chacun des lots ont été comptés et la longueur (totale) de tous les survivants a été mesurée. Par ailleurs les survivants des lots 2 et 4 ont été passés au détecteur de marques afin d'évaluer le taux de rétention de marques sur les survivants après 1 mois.

Après avoir constaté le très faible taux de rétention des marques sur les ombles de très petite taille (groupe A), la dynamique des pertes de marque a été suivie sur un lot d'ombles issus du groupe B. Le contrôle du taux de rétention a été effectué régulièrement jusqu'à ce que l'on ne constate plus de pertes de marque. A chaque contrôle tous les poissons du lot ont été passés au détecteur de terrain. Les marqués et non marqués ont été comptés à chaque contrôle.

\subsection{Essais de 1986}

Après avoir pris directement contact sur place avec les ingénieurs de N.M.T., nous avons modifié la procédure de réglage de la machine. La position de la marque dans l'aiguille au moment de la rétraction de cette dernière a été réglée en position terminale. Par ailleurs, les marques ont été implantées plus profondément c'est-à-dire dans le cartilage situé juste en arrière de la ligne (fictive) joignant les 2 cavités olfactives (position P, fig. 1b). En 1985 les marques avaient été implantées juste en avant des cavités olfactives (fig. $1 \mathrm{~b}$ ).

L'essai principal a porté sur un groupe d'omble (A') de très petite taille $(\bar{L}=28.2 \mathrm{~mm} ; 22$ à 35$)$ voisine de celle du groupe A de 1985. L'essai visait à comparer la croissance et la survie après 1 mois entre des témoins non marqués ( 3 lots de 300 ) et des individus marqués magnétiquement ( 3 lots de $300)$. Les six lots ont été élevés en bacs séparés. La dynamique de perte de marque a été suivie sur deux lots de 300 ombles marqués: un lot d'ombles de très petite taille, issu du groupe $A^{\prime}$ et un lot d'ombles de petite taille, voisine de celle du groupe B marqué en 1985.

Les données de 1985-86 ont été traitées à l'aide de tests statistiques classiques ( $X 2$, $t$ et $F$ ).

\section{II - RÉsultats}

\section{Vitesse et coûts du marquage}

Après entraînement, la vitesse de marquage magnétique a été de 250-300/heure/personne pour les ombles les plus petits $(2-3 \mathrm{~cm})$ et de $400-500 /$ heure/personne pour les plus gros $(4-5 \mathrm{~cm})$. Ces vitesses ont été calculées dans le cas d'une seule et même personne effectuant l'ensemble des opérations (fig. 2 b) et sans compter le travail préliminaire (fig. 2a). Le tableau 2 donne une évaluation

\begin{tabular}{|l|l|c|c|}
\hline Type de marquage & Taille & $\begin{array}{c}\text { Vitesse } \\
\text { nombre par } \\
\text { marqueur et } \\
\text { par heure }\end{array}$ & $\begin{array}{c}\text { Coût par } \\
\text { poisson marqué } \\
\text { en F. }\end{array}$ \\
\hline \multirow{2}{*}{ Magnétique (1/2 M) } & $25-35$ & $250-300$ & $0,48-0,51$ \\
\cline { 2 - 4 } & $35-55$ & $300-500$ & $0,43-0,48$ \\
\hline \multirow{2}{*}{$\begin{array}{l}\text { Magnétique (1/2 M) } \\
\text { et }\end{array}$} & $25-35$ & $250-300$ & $0,55-0,59$ \\
\cline { 2 - 5 } & $35-55$ & $300-500$ & $0,50-0,56$ \\
\hline $\begin{array}{l}\text { Cautérisation de } \\
\text { adipeuse (ad }\end{array}$ & $25-55$ & $500-600$ & $0,07-0,08$ \\
\hline $\begin{array}{l}\text { Ablation des } \\
\text { pelviennes (2pV }\end{array}$ & $25-55$ & $300-400$ & $0,10-0,13$ \\
\hline \multirow{2}{*}{\begin{tabular}{l} 
ad et 2 pV \\
\hline
\end{tabular}} & $25-55$ & $190-240$ & $0,17-0,21$ \\
\hline
\end{tabular}

Tableau 2 : Evaluation des vitesses et des coûts de marquage dans le cas des petits (25-55 mm)salmonidés.

Table 2 : Evaluation of rate and costs of marking small $(25-55 \mathrm{~mm})$ salmonids. 
des coûts de marquage en prenant comme coût de main-d'œuvre: $40 \mathrm{~F} /$ personne/heure. Pour le marquage magnétique le coût du marquage inclut le prix de la marque $(0,35 \mathrm{~F}$ pièce) mais non l'amortissement de la machine. Dans les conditions de l'expérimentation réalisée, le coût du marquage magnétique de petits salmonidés avec des demi-marques codées varie de 40 à 60 centimes selon la taille et que l'on réalise ou non l'ablation de la nageoire adipeuse (tab. 2). Notons cependant que ces coûts sont susceptibles de fluctuer en fonction des conditions propres à chaque campagne de marquage.

\section{Rétention des marques et suivi de l'influence du marquage sur la croissance et la survie}

\subsection{Essais de 1985, Ombles de très petite taille}

\section{- Taux de rétention :}

Lors des premiers essais sur les ombles de très petite taille $(\mathrm{L}=25,5 \mathrm{~mm} ; \overline{\mathrm{L}}: 22$ à $30 \mathrm{~mm})$ le taux de réteption des marques, mesuré à 1 mois, a été faible, voisin de $18 \%$ (tab. 1 ).

- Survie :

Comme dans le cas de tous les essais, les mortalités en cours de marquage ont été très faibles $(<0,5 \%)$. Le taux de survie après 1 mois des ombles marqués magnétiquement $(1 / 2 \mathrm{M})$ est élevé $(96 \%)$ et non significativement $(\alpha=5 \%)$ différent de celui des ombles marqués par cautérisation de l'adipeuse (ad $\phi ;$ tab. 1). Le taux de survie des ombles doublement marqués (1/2 M et ad $\phi)$ est voisin $(82,0 \%)$ mais légèrement inférieur $(\alpha=5 \%)$ à celui $(88,3 \%)$ des témoins non marqués.

\section{- Croissance en longueur :}

La taille moyenne à 1 mois des individus marqués magnétiquement n'est pas significativement $(\alpha=5 \%)$ différente de celle des ombles à adipeuse cautérisée. La taille moyenne du lot d'ombles doublement marqués $(1 / 2 M+$ ad $\phi)$ est légèrement mais significativement $(\alpha<1 \%)$ supérieure $(+2 \mathrm{~mm})$ à celle des témoins non marqués (tab. 1). La différence n'est cependant plus significative $(\alpha=5 \%)$ lorsque l'on considère uniquement les survivants ayant gardé leur marque.

\section{2. - Essais de 1985. Ombles de petite taille}

\section{- Taux de rétention :}

Lors des premiers essais, en 1985 , le taux de rétention a été nettement plus élevé $\{69,5$ $-79,7 \%$ ) dans le cas des ombles de plus grande taille initiale $\bar{L}=46,6 \mathrm{~mm} ; \mathrm{L}: 35$ à $55 \mathrm{~mm}$ ). Les pertes de marque se sont étalées sur 5 semaines mais la moitié d’entre elles ont eu lieu lors de la première semaine (fig. 3 ).

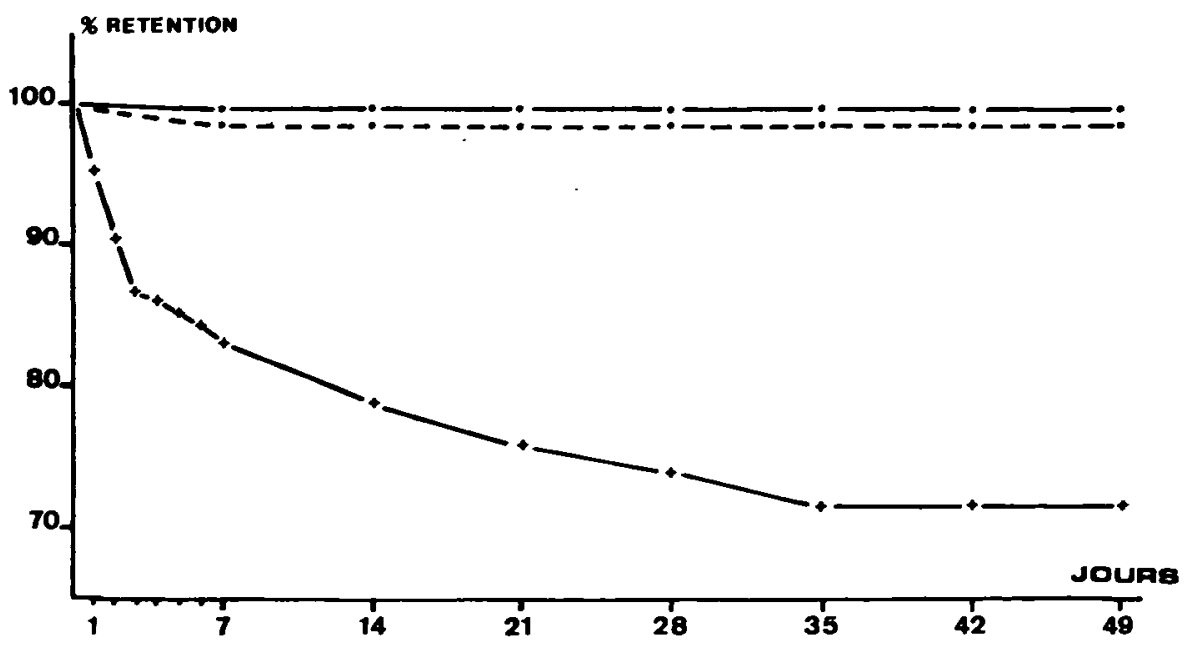

Figure 3 : Suivi du taux de rétention des marques en fonction du temps
$1 \mathrm{x}$
X) lot $B$ en $1985 ;\left(.-.-\right.$ ) lot $A^{\prime}$ en $1986 ;$ (.
lot $B^{\prime}$ en 1986 .

Figure 3 : Evolution of tag retention

$\left(x \_x\right)$ batch $B$ in $1985:(. \ldots)$ batch $A^{\prime}$ in 1986; $(\ldots \ldots)$ batch $B^{\prime}$ in 1986. 


\section{- Survie :}

Le taux de survie après 1 mois est élevé $(92,0-95,0 \%)$ dans tous les lots (tab. 1) et il n'apparaît aucune différence significative $(\alpha=5 \%)$ entre les deux lots d'un même bac (tab. 1).

\section{- Croissance en longueur :}

Les individus marqués magnétiquement $(1 / 2 M)$ ont une taille moyenne légèrement $(+1 \mathrm{~mm})$ mais significativement ( $\alpha=5 \%$ ) supérieure à celle des ombles dont l'adipeuse a été cautérisée (tab. 1). II n'apparaît pas de différence significative $(\alpha=5 \%)$ entre le lot doublement marqué $(1 / 2 \mathrm{M}$ et ad $\phi)$ et les témoins non marqués. Les observations précédentes sont les mêmes à la fois lorsque l'on prend en compte soit tous les survivants des lots marqués magnétiquement, soit seulement ceux ayant gardé leur marque.

\subsection{Essais de 1986}

\section{- Taux de rétention :}

Les modifications du réglage de la machine et l'implantation plus profonde des marques ont permis de fortement augmenter le taux de rétention des marques, à la fois dans le cas des ombles de très petite taille $(98,0-98,3 \%)$ et dans celui des ombles de petite taille $(99,7 \%)$. Dans les deux cas, les pertes de marques ont été constatées dans la première journée suivant le marquage alors qu'aucune perte n'a été décelée lors des 7 semaines suivantes (fig. 3 ).

- Survie des ombles de très petite taille :

Que les ombles soient marqués magnétiquement ou non, les taux de survie à 1 mois sont élevés $(94,7 \%$ à $98,3 \%)$. II n'apparaît aucune différence significative $(\alpha=5 \%)$ soit sur l'ensemble des 6 lots, soit entre lots marqués, soit entre lots non marqués, soit entre l'ensemble des marqués et des non marqués (tab. 1).

\section{- Croissance en longueur des ombles de très petite taille :}

La figure 4 précise la structure de taille après 1 mois pour l'ensemble des survivants marqués et l'ensemble des témoins non marqués. La structure de taille varie entre bacs témoins mais non entre bacs de marqués. La longueur moyenne n'est pas significativement $(\alpha=5 \%)$ différente entre les bacs de marqués $(1 / 2 \mathrm{M})$ et les bacs témoins d'ombles non marqués. Elle varie selon les bacs entre 39,2 et $40,6 \mathrm{~mm}$ pour les non marqués et entre 39,5 et $40,3 \mathrm{~mm}$ pour les ombles marqués magnétiquement (tab. 1).
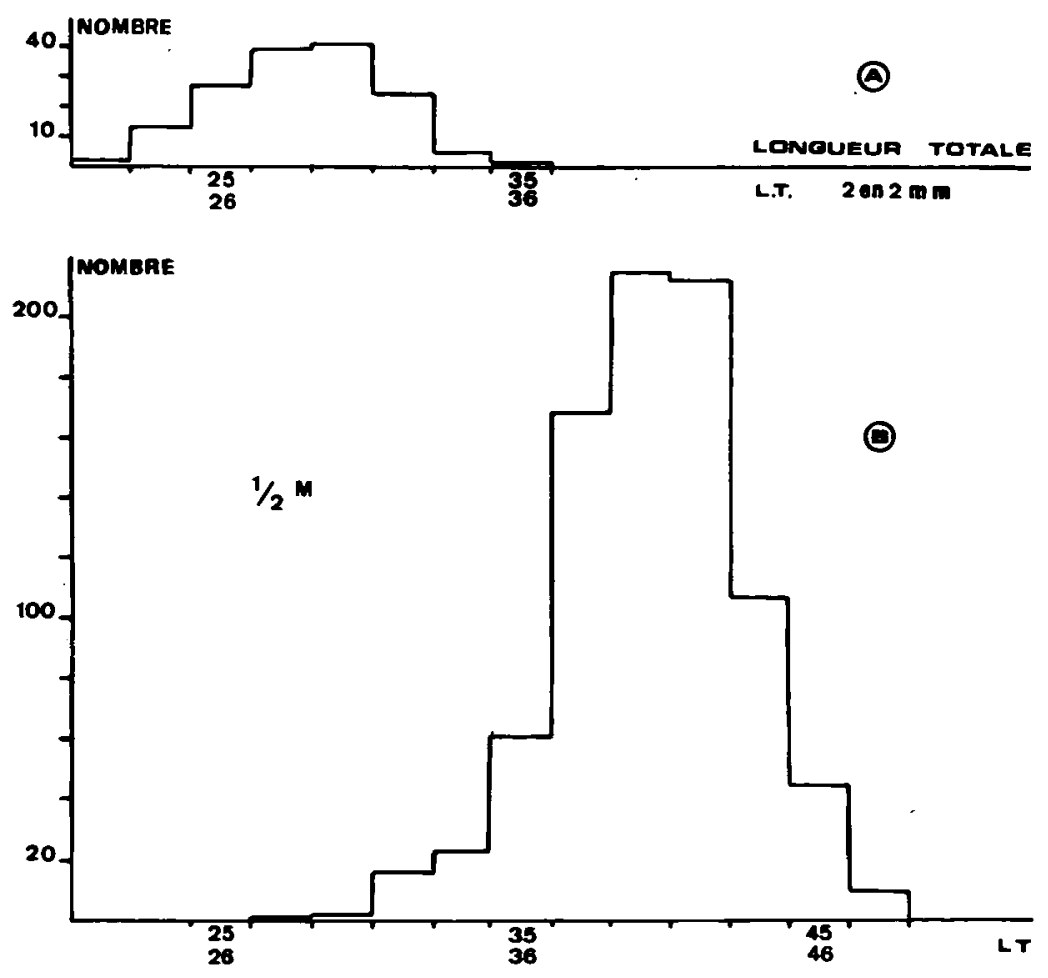


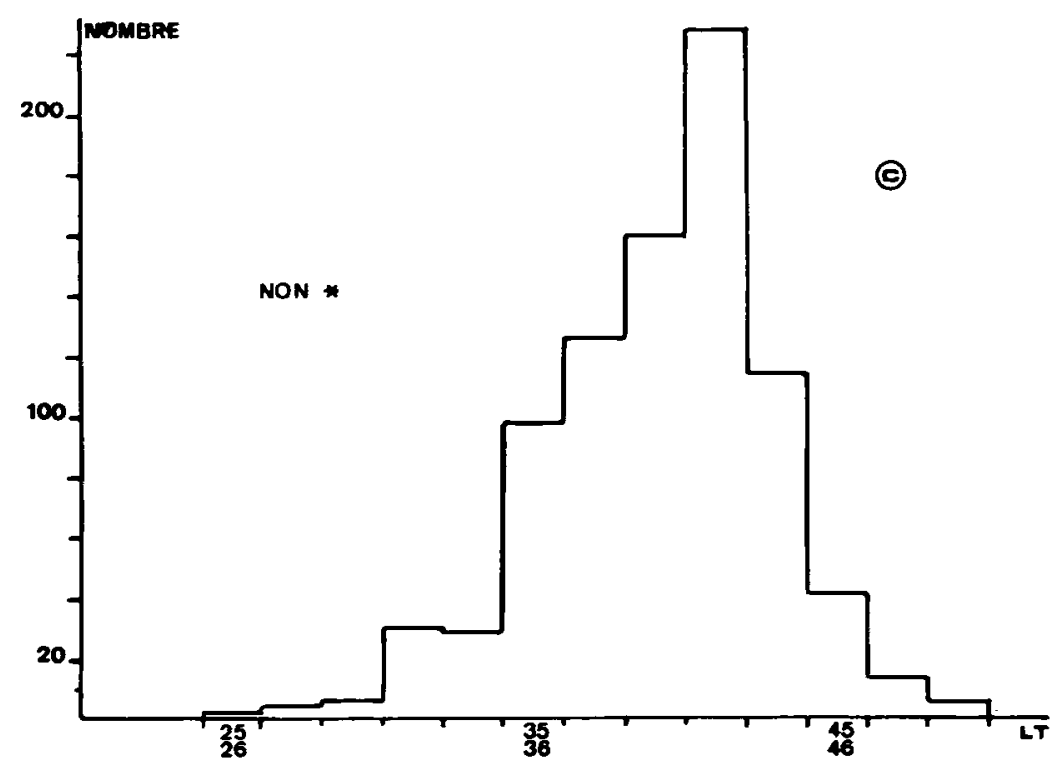

Figure 4 : Structure de taille $(2 \mathrm{en} 2 \mathrm{~mm}$ ) des ombles après un mois d'élevage. (Expérience de 1986).
A) Taille initiale au moment du marquage (mesures sur 150 alevins)
B) Ensemble des survivants marqués magnétiquement
C) Ensemble des témoins non marqués.

Figure 4 : Size structure of charrs after 1 month of rearing (Experiment of 1986)
A) Initial size structure just before tagging (sample of $150 \mathrm{fry}$ )
B) Totality of survivors with magnetic tags
C) Totality of charrs without tag (control batch)

\section{DISCUSSION}

Le système de marquage magnétique avec des marques entières $(I=1 \mathrm{~mm})$ n'est généralement utilisé que pour des poissons dépassant $1,8-2 \mathrm{~g}$ à cause des contraintes associées à la taille de la marque et à son implantation optimale dans le cartilage nasal (OPDYCKE et ZAJAC, 1981). MENG et al. (1986) recommandent pour le système marque entière de ne pas l'utiliser sur des corégones de moins de $60 \mathrm{~mm}$, ceci afin de permettre une implantation profonde ( $>2,5 \mathrm{~mm}$ ) et de minimiser les problèmes de pertes de marques et de mortalité liée au marquage. Les auteurs indiquent, pour ces corégones de $60 \mathrm{~mm}$ marqués et suivis en élevage pendant 10-15 j, un taux de mortalité de $5 \%$ lié au marquage et $\mathbf{8 , 5} \%$ de pertes de marques.

Le système de marquage magnétique avec des demi-marques a été développé à l'origine pour permettre le marquage de juvéniles de saumon du Pacifique. Le fabricant préconise les demi-marques pour des poissons de moins de $2 \mathrm{~g}$, en précisant néanmoins que le poids minimum recommandé est de $250 \mathrm{mg}$. Les essais décrits dans la présente étude portent donc sur des juvéniles appartenant à la partie inférieure de cette gamme et même en deçà puisque un lot de $28 \mathrm{~mm}$ de longueur moyenne $(P=$ $0.2 \mathrm{~g})$ a été marqué avec succès. THROWER et SMOKER (1984) dans le cas d'alevins de saumon pink $(L: 27-38 \mathrm{~mm} ; \bar{L}=33 \mathrm{~mm} ; P=250 \mathrm{mg}$ ) ont obtenu de bons résultats avec le système demi-marque: survie de $99,8 \%$ et rétention de $95,7 \%$ après $2-9$ jours en élevage.

Lors des essais de 1985 la vitesse de marquage de gros $(8-10 \mathrm{~cm})$ juvéniles d'omble chevalier (données non publiées) a été nettement plus élevée $(600-800 / \mathrm{h})$ que celle observée $(250-450 / \mathrm{h}) \mathrm{sur}$ les petits individus $(25-55 \mathrm{~mm})$. THROWER et SMOKER (1984) indiquent, dans le cas d'alevins de saumons de $3-4 \mathrm{~cm}$, une vitesse moyenne de $350 / \mathrm{h}$ et une vitesse de pointe approchant $600 / \mathrm{h}$. LEARY et MURPHY (1975) indiquent une vitesse de $300 / \mathrm{h}$ dans le cas d'anchois de $4-5 \mathrm{~cm}$.

Un des critères importants de succès d'une opération de marquage magnétique est l'obtention d'un taux élevé de rétention des marques. Il est donc nécessaire de maîtriser les facteurs permettant d'optimiser la rétention des marques. OPDYCKE et ZAJAC (1981) indiquent que, dans des essais de marquage magnétique nasal (1/2 marques) de juvéniles de saumon chum de $0,8 \mathrm{~g}$, le taux de rétention des marques a varié de 66 à $98 \%$ en fonction de l'expérience des marqueurs. La présente 
étude, en accord avec THROWER et SMOKER (1984), montre que l'obtention d'un bon taux de rétention des marques est en grande partie conditionnée par:

- la pratique du marquage sur des lots homogènes avec l'utilisation d'un embout bien adapté à la morphologie de la tête des poissons marqués et l'utilisation d'une aiguille très pointue,

- l'optimisation du réglage fin de l'injecteur de marque (position terminale de la marque lors du retrait de l'aiguille) et du positionnement de la marque dans la tête,

- le contrôle régulier de l'implantation des marques et du fonctionnement de l'appareil assurant la magnétisation et le contrôle du marquage.

Le contrôle de l'implantation des marques est le plus souvent effectué par dissection. Cependant dans le cas des petits corégones $(3-4 \mathrm{~cm})$ la marque peut être directement visible par transparence. L'implantation des marques est généralement réalisée dans le cartilage situé juste en avant des cavités olfactives. Dans le cas de la présente étude, et pour les très petits ombles, seule une implantation plus profonde (juste en arrière des cavités olfactives) a permis une bonne rétention des demi-marques. Cette observation rejoint celles de THROWER et SMOKER (1984) qui indiquent que, dans le cas des alevins de saumon pink, la masse cartilagineuse en avant des cavités olfactives est trop réduite pour permettre une bonne rétention des demi-marques, d'où la nécessité d'une implantation plus profonde.

Plusieurs auteurs (ISAKSSON et BERGMAN, 1977; OPDYCKE et ZAJAC 1981 ; THROWER et SMOKER, 1984 ; MENG et al., 1986) signalent la faiblesse du taux de mortalité (<5\%) sur des lots de poissons marqués magnétiquement et stabulés de quelques jours à quelques semaines. La présente étude, réalisée en comparaison avec des témoins non marqués ou marqués par ablation de l'adipeuse, renforce cette tendance. Mis à part le cas des paramètres survie et croissance, l'influence du marquage magnétique reste encore peu étudiée. En l'absence d'études poussées, il n'est pas possible d'avancer, dans le cas des très petits salmonidés, que le marquage magnétique n'a aucune influence sur le système olfactif. Notons cependant que, selon THROWER et SMOKER (1984), le taux de retour de juvéniles de saumons pink marqués à l'émergence est voisin de celui des non marqués. JEFFERTS et al. (1963) puis BERGMAN et al. (1968), en accord avec la présente étude, n'ont pas noté d'influénce du marquage magnétique sur la croissance. Pour suivre les opérations de repeuplement, la marquage magnétique des salmonidés est souvent associé à une ablation de l'adipeuse afin de permettre un premier repérage externe des poissons marqués. Pour les très petits individus, l'ablation de l'adipeuse est réalisable par cautérisation (CHAMPIGNEULLE et ESCOMEL, 1984) mais peut introduire une légère baisse du taux de survie si elle est pratiquée sur des juvéniles de taille inférieure à $33 \mathrm{~mm}$. D'après les résultats obtenus dans la présente étude, la pratique du double marquage $(1 / 2 \mathrm{M}+\mathrm{ad} \phi)$ n'influence pas significativement la survie et la croissance dans le cas des petits (35-55 mm) ombles. Cette pratique est donc utilisable lorsque l'on veut faciliter le suivi des recaptures et /ou évaluer le taux de rétention des marques magnétiques.

\section{CONCLUSION - RECOMMANDATIONS}

Les expériences effectuées ont montré que le marquage magnétique nasal, pratiqué avec des demi-marques codées, s'avère performant sur de très petits (à partir de $25 \mathrm{~mm}$ ) salmonidés lorsque la technique est bien maîtrisée. Il apparaît donc possible, sous réserve d'un fort taux de rétention. d'identifier, avec un minimum de biais liés au marquage, de nombreux lots de juvéniles de salmonidés. Le marquage apparaît réalisable dès le début de la phase d'alimentation ou même en fin de résorption de vésicule dans le cas des gros alevins. Notons cependant que des études restent à faire pour préciser les dommages éventuels causés par la nécessité de pratiquer une implantation profonde des demimarques.

Pour chaque lot marqué il est hautement souhaitable de suivre (mortalité, dynamique de pertes de marques), en pisciculture, un sous-lot témoin. Dans le cas de repeuplements ou d'études fines où l'on désire minimiser les biais liés aux pertes de marques, la procédure suivante peut être préconisée: stabulation du lot marqué jusqu'à annulation des pertes de marques dans un sous-lot témoin puis contrôle du marquage sur le lot entier avec élimination des non-marqués. Lorsque l'expérience ou le relâcher ne peuvent être différés, le suivi du sous-lot témoin gardé permet l'évaluation du taux de perte de marques.

La pratique du double marquage $(1 / 2 \mathrm{M}+$ ad $\phi)$ offre une possibilité complémentaire d'évaluer la rétention des marques sur les poissons relâchés.

\section{REMERCIEMENTS :}

Ce travail entre dans le cadre d'une convention INRA-CSP ( $n^{\circ} 83-624$ du 6/12/83). 


\section{BIBLIOGRAPHIE}

BERGMAN P.K., JEFFERTS K.B., FISCUS H.F. and HAGER R.C., 1968. A preliminary evaluation of an implanted coded wire fish tag. Washington Dep. of Fisheries, Fisheries Res. Papers 3(1), 63-84.

CHAMPIGNEULLE A. et ESCOMELJ., 1984. Note technique. Marquage des salmonidés de petite taille par ablation de l'adipeuse ou des nageoires pelviennes. Bull. Fr. Piscic., 293-294, 52-58.

CRISTAU-OUOST I., 1980. Essais d'étude comparative de différents types de marquage de poissons. Observations histologiques préliminaires de l'effet du cryomarquage. Thèse de Doctorat de Troisième Cycle. Univ. Claude Bernard, Lyon I, 1 vol., 201 p.

DUMAS J. et PROUZET P., 1982. Marquage magnétique interne : essais de tolérance par de jeunes saumons atlantiques (Salmo salar L.). Bull. Fr. Piscic., 287, 23-33.

HAGER B., 1975. A technique for mass recovery of wire tags from young salmonids. Prog. Fish. Cu/t., $37,51$.

HARACHE Y., LAGARDE P. et PROUZET P., 1978. Essais d'une marque magnétique interne pour smolts de saumon. Pisc. Fr., 53-54, 81-86.

HARGREAVES N.B. and LE BRASSEUR R.J., 1986. Size selectivity of Coho (Ancorhynchus kisutch) preying on juvenile chum Salmon (O. keta). Can. J. Fish Aquat. Sci., 43, 581-586.

ISAKSSON A. and BERGMAN P.K., 1977. Evaluation of two tagging methods used to study the efficiency of atlantic salmon hatchery operation in Iceland. ICES C.M. 1977/M:36.

JEFFERTS K.B., BERGMAN P.K. and FISCUS H. F., 1963. A coded wire identification system for macro-organisms. Nature, 198, 460-462.

LEARY D.F. and MURPHY G.I. 1975. A successful method for tagging the small, fragile engraulid (Stolephorus purpureus). Trans. Am. Fish. Soc., 104, 53-55.

MENG H.J., MULLER R. and GEIGER W., 1986. Growth, mortality and yield of stocked coregonid fingerlings identified by microtags. Arch. Hydrobiol. Beih. Ergebn. Limnol., 22, 319-325.

OPDYCKE J.D. and ZAJAC P., 1981. Evaluation of half-length linary coded wire tag application in juvenile chum salmon. Prog. Fish. Cult., 43, 48.

SALOJARVI K., 1986. Review of whitefish (Coregonus lavaretus $L$.) fingerling rearing and stocking in Finland. Arch. Hydrobiol. Beih. Ergebn. Limnol., 22, 29-144.

THROWER F.P., SMOKER W.W., 1984. First adult return of Pink Salmon tagged as emergents with binary-coded wires. Trans. Am. Fish. Soc., 113, 803-804. 